\title{
Environmental assessment of an offshore infrastructure using a beltline of concrete caissons and impact of logistical decisions
}

\author{
Juliette LANGLOIS ${ }^{1,2}$, Guillaume JUNQUA ${ }^{2,3}$, \\ Jean-Claude SOUCHE ${ }^{4}$, Sylvain PIOCH ${ }^{1}$
}

1. CEFE, UMR5175, CNRS - Université de Montpellier, Université Paul-Valéry Montpellier 3 - EPHE, Montpellier Cedex 5, France.

juliette.langlois84@gmail.com

2. Elsa, Research group for Environmental LifeCycle and Sustainability Assessment, Montpellier, France.

3. LGEI, IMT Mines Ales, Ales, France.

4. LMGC, IMT Mines Ales, Univ Montpellier, CNRS, Ales, France.

\begin{abstract}
:
Mainly due to the attraction of the coastal areas, projects encroaching on the sea are growing in France and worldwide. Besides risk management (storms and flooding), coastal erosion and biodiversity loss, the issue of matter and energy consumption appears of importance for such infrastructures, inducing environmental impacts at a global scale. This study aims at quantifying these environmental impacts, using Life Cycle Assessment (LCA), applied to an offshore infrastructure using a beltline of concrete caissons. This technology has been used in Monaco, for the Anse du Portier's extension, because it ensured a good wave resistance, a cost reduction (works in open sea being limited) and a soil level being in accordance with architectural landscape constraints and sea view. A realistic case study has been used to scale the assessment, suggesting optimal solutions based on the current knowledge. The main construction steps inducing environmental impacts as well as the opportunities of technical improvements allowing their reduction have been identified and characterized. Furthermore, effects of logistical decisions (for transportation distances and sourcing) on the global performances have been quantified. Results highlight the importance of supply chain choices on global performances. Territorial constraints can reduce logistical options, due to local deposit size and availability, time constraints for the construction, storage and loading capabilities on site. These limitations are discussed as well as the environmental concern integration in the project management.
\end{abstract}

Mots-clés :

Extension en mer, Analyse de Cycle de Vie (ACV), Performances environnementales, Ecoconception, Logistique, Ceinture de caissons. 


\section{Thème 4 - Ouvrages portuaires, offshore et de plaisance}

\section{Introduction}

Les littoraux font aujourd'hui face à une artificialisation terrestre croissante du fait de leur forte attractivité. Avec près de $40 \%$ de la population mondiale vivant à moins de 100 $\mathrm{km}$ d'un environnement côtier (MEA, 2005), les projets d'îles artificielles ou d'exondation en mer se multiplient en France et dans le monde. Ces aménagements côtiers soulèvent à la fois la question de la gestion des risques (tempêtes et submersion) mais aussi celle de l'érosion du littoral et de sa biodiversité, et plus globalement celle d'une consommation de matière et d'énergie importantes, avec son lot d'impacts sur l'environnement à une échelle globale. Les espaces littoraux étant parmi les plus vulnérables et les plus menacés, mais aussi les plus riches en termes de biodiversité et services rendus à l'homme (MEA, 2005), de récentes recommandations appellent à la relocalisation de certaines activités vers l'intérieur des terres (MINEO-KLEINER \& MEUR-FEREC, 2016), cela afin d'atténuer ces risques et les pressions subies par les écosystèmes côtiers. Pourtant, la tendance actuelle est inverse. Depuis l'Antiquité, 5241 ha et 78.5 ha d'aménagements ont été gagnés respectivement sur les côtes françaises et monégasques (MEINESZ et al., 2013), tendance encore plus flagrante dans les villesétats, qui ne peuvent s'étendre que sur la mer du fait de leurs frontières.

Parmi les solutions techniques envisageables pour des extensions sur la mer, le terre-plein avec digue d'enclôture en caissons en béton armé présente de nombreux avantages : protection de l'ouvrage contre la houle et atténuation des bruits solidiens par des chambres Jarlan, possibilité de travailler abrité de la mer une fois la ceinture de caissons posée, emprise limitée sur les fonds contrairement à un remblai à talus. Cette technique limite également les contraintes de temps et/ou d'argent car elle limite les travaux en mer ouverte et car sa maintenance est simple durant l'exploitation. La commercialisation et l'assurabilité des superstructures sont aisées car la technique est éprouvée et la distinction est claire entre l'ouvrage maritime et les structures porteuses des bâtiments. Ces avantages ont conduit au choix de la technique d'une digue en caissons en béton armé à Tanger comme pour le terre-plein de Fontvieille et pour la récente extension de l'Anse du Portier à Monaco.

D'un point de vue environnemental, les enjeux sont de taille, du fait de la taille des projets et des besoins en matériaux et en énergie qu'ils suscitent. De plus, ils sont construits en générant une destruction inévitable et importante des petits fonds marins.

La présente étude a pour but d'identifier des points clés d'écoconception pour une extension sur la mer par ceinture de caissons. L'Analyse de Cycle de Vie (ACV) environnementale est utilisée, en tant que méthode robuste, normalisée (ISO 14044, 2006) et usuelle d'évaluation des impacts et comme outil d'écoconception. La méthode ACV permet d'évaluer les impacts environnementaux de façon exhaustive, tout au long du cycle de vie de l'objet étudié. La partie 2 détaille le cadre méthodologique et les hypothèses de travail. Le dimensionnement se base sur les contraintes géophysiques d'une extension par terre-plein et digue d'enclôture en caissons en béton armé située sur 


\section{XVIèmes Journées Nationales Génie Côtier - Génie Civil \\ Le Havre 2020}

la masse d'eau de Menton à Nice. Les choix technologiques et logistiques de deux scénarios sont proposés : l'un dont les critères environnementaux sont optimisés, en s'appuyant sur des techniques connues et réalisables à échelle industrielle, et un autre correspondant aux pratiques courantes du secteur. Les résultats de l'analyse de contribution ainsi que l'impact de choix logistiques d'approvisionnement sur les performances environnementales d'une telle extension en mer sont fournis en partie 3. Les limites de l'étude, notamment en terme d'applicabilité de solutions plus performantes sont abordées en partie 4 .

\section{Méthode : définition des deux scénarios étudiés et données d'inventaire}

L'objectif de l'étude est d'identifier des paramètres logistiques clés influençant les performances environnementales d'une extension construite sur la mer, à l'aide d'une digue de caissons en béton armé disposant de chambres Jarlan, d'un terre-plein en remblais et de pieux de fondation pour les superstructures à usage d'habitation. Le dimensionnement de l'extension se base sur les conditions géophysiques de la masse d'eau de Menton à Nice, voir schéma Figure 1 (ARISTAGHES et al., 2018). L'extension s'étend sur 6 ha, avec une profondeur moyenne des fonds de $35 \mathrm{~m}$ en périphérie de l'infrastructure. Les houles sont de direction Nord-Ouest et la houle centennale d'une durée de 3 heures est majorée de $10 \%$, ce qui implique de résister à une hauteur de houle de 5,03 m. Les ouvrages sont en zone de sismicité 4. La durée d'utilisation prévue est de 200 ans (dont 100 ans garantis sans maintenance). L'unité fonctionnelle choisie reprend ces caractéristiques : réaliser une extension en mer de 6 ha, pour une durée de vie de 200 ans. L'évaluation environnementale est effectuée avec le logiciel SimaPro 8.5.0.0, avec les bases de données EcoInvent 3 et la méthode ILCD 2011, recommandée par la Commission Européenne dans le cadre de l'ACV.

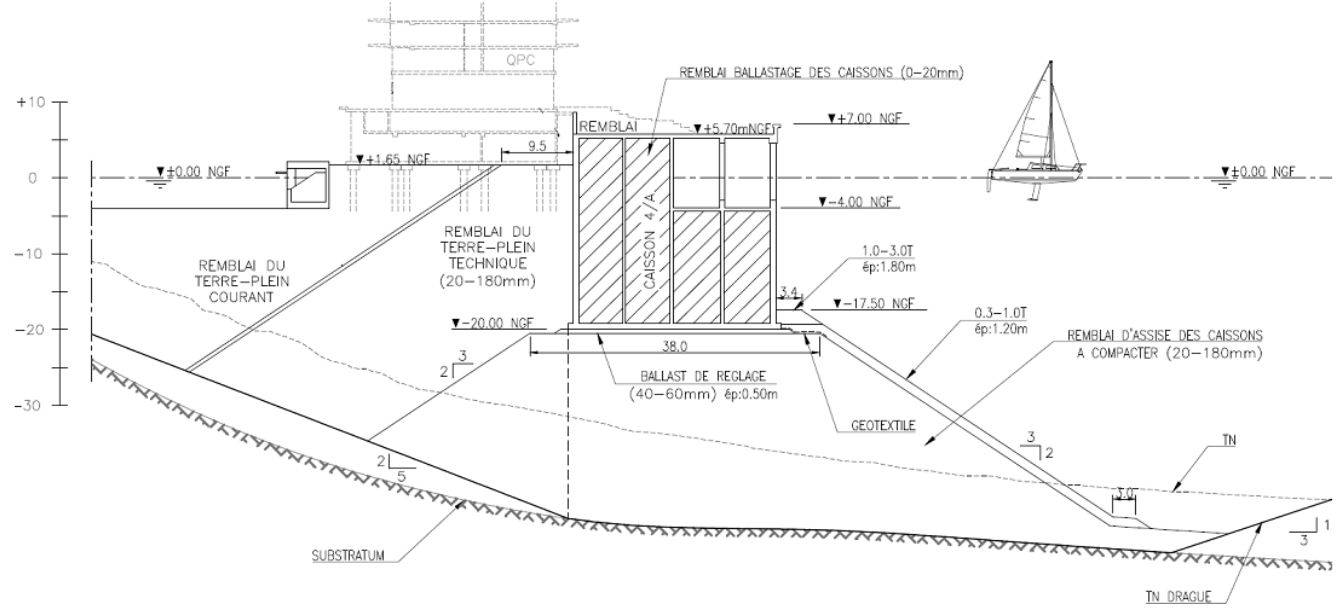

Figure 1. Coupe transversale d'un caisson, des remblais et des superstructures d'une extension par ceinture de caissons (ARISTAGHES et al., 2018). 


\section{Thème 4 - Ouvrages portuaires, offshore et de plaisance}

Sur la base de cet inventaire, deux scénarios ont été étudiés : le scénario 1, faisant l'objet d'une optimisation sur les critères environnementaux au vu des connaissances techniques actuelles, et le scénario 2, qui tient compte de l'ensemble des contraintes techniques et politiques du projet. Ainsi, on a choisi pour le scénario 1 des distances optimisées pour l'approvisionnement des matériaux, l'utilisation de matériaux de substitution partielle du ciment dans le béton des pieux et des caissons, le lavage des matériaux de remblai à l'eau de mer après transport et matériaux avec moins de fines pour limiter la turbidité. Sur la base de ces hypothèses, pour chaque étape de la construction (voir schéma des procédés Figure 2), on fournit les consommations de matières, d'énergie et les distances d'approvisionnement (en bleu), les principales émissions vers l'environnement (en rouge) et les hypothèses d'écoconception (en vert) du scénario 1.

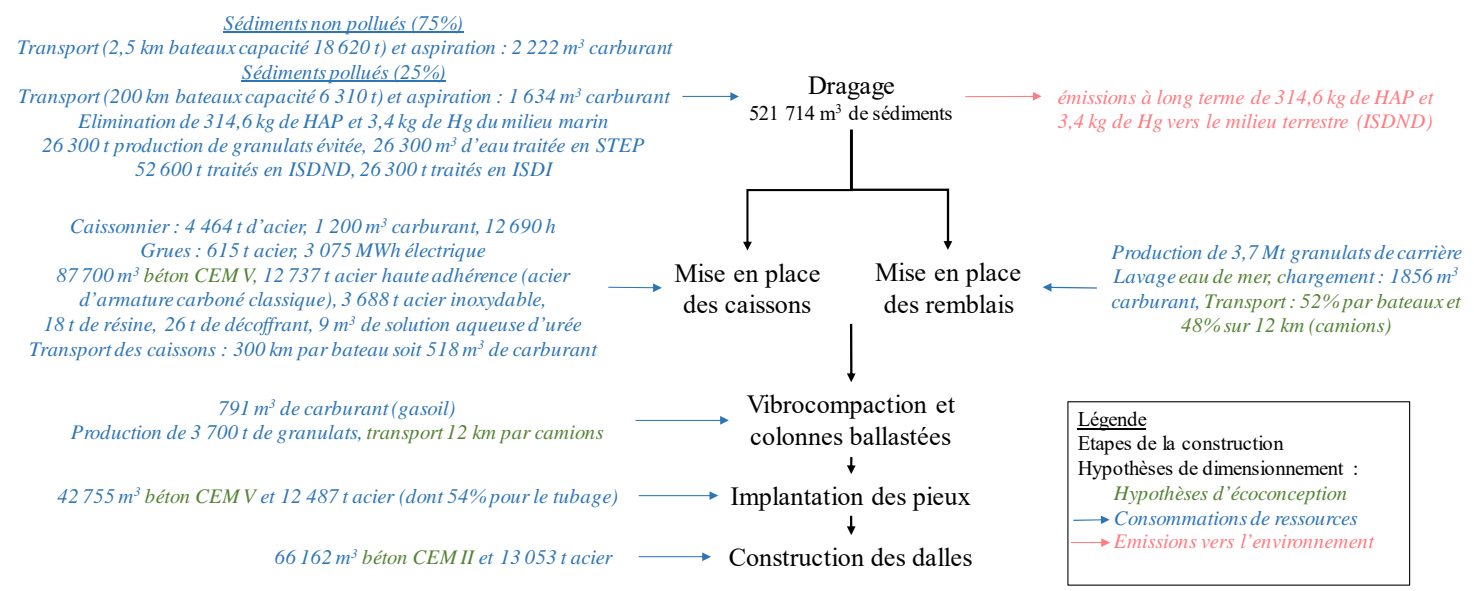

Figure 2. Schéma des procédés et principales données d'inventaire du scénario 1.

Le dragage vise à supprimer les matériaux compressibles sous les futurs remblais afin d'assurer leur stabilité et des tassements compatibles avec les usages du terre-plein. Les produits de dragages comprennent $75 \%$ de sédiments non pollués, aspirés par une drague puis rejetés en mer $2 \mathrm{~km}$ plus loin et $25 \%$ de sédiments pollués qui ont été traités à terre, dans un centre situé à $200 \mathrm{~km}$, via un séchage par percolation.

Les 18 caissons de la digue d'enclôture du terre-plein ont été fabriqués dans un caissonnier dans le port industriel de Marseille, offrant un tirant d'eau suffisant. La mise en place des caissons sur leur place définitive a nécessité la mise en place préalable d'une couche de pose en matériaux de carrières assurant la couche de fondation des caissons. Les remblais utilisés représentent de très grands volumes : 1,5 Mt de remblais d'assise des caissons, 0,9 Mt de remblais de terre-plein technique à l'arrière des caissons. Les remblais hydrauliques et de remplissage des caissons représentent respectivement 0,77 et $0,38 \mathrm{Mt}$ de granulats non portants. 


\section{XVI'̀mes Journées Nationales Génie Côtier - Génie Civil \\ Le Havre 2020}

Des inclusions par vibrocompaction et la mise en place des colonnes ballastées assurent la stabilité des remblais. Les pieux de fondation des futurs bâtiments, peuvent alors être réalisés ainsi que les dalles de fondation.

Dans le scénario 1, on choisit un scénario d'approvisionnement optimisé en granulats. Cette hypothèse suppose d'une part la proximité immédiate de carrières et d'autre part l'autorisation d'exploitation suffisante pour garantir à la fois le marché local usuel et le projet d'extension. Un scénario d'apport local de matériaux a ainsi été proposé, sur la base des capacités de production nominale des carrières de la Turbie : $2 \mathrm{Mt}$ par an, dont 0,8 Mt couvrant les besoins du secteur (BRGM, 2000). Ainsi, 1,2 Mt par an pourraient être approvisionnés localement. Compte tenu du relief escarpé de ces carrières, le stockage de matériaux sur ces sites ou dans l'arrière-pays apparaît comme marginal.

Dans ce type de projets, il est usuel d'assurer un approvisionnement par bateau de grande capacité (35000 t), depuis les ports voisins (250 à $300 \mathrm{~km}$ suite à un transport par camion

de 10 à $20 \mathrm{~km}$ ). Ces hypothèses seront modélisées dans un deuxième scénario (scénario 2, non optimisé) qui permet de diversifier les approvisionnements et ainsi de limiter la circulation des camions sur place. De plus, ce scénario permet de s'approvisionner rapidement et en très grande quantité, depuis des sites où d'importantes quantités sont disponibles et stockées. Dans ce second scénario, on suppose que les ciments des bétons ne sont pas partiellement substitués et que le lavage des matériaux de carrière est effectué à l'eau douce avant chargement des camions.

En se basant sur la temporalité des opérations de remblaiement pratiquées par bateau, et servant de base pour définir un délai de chantier acceptable, dans le scénario 1 les remblais d'assise des caissons, remblais techniques et remblais hydrauliques pourraient être produits localement, respectivement à hauteur de $37 \%, 100 \%$ et $29 \%$, soit une moyenne de $48 \%$ d'approvisionnement local et 52\% d'approvisionnement par bateau.

Les performances environnementales des deux scénarios sont présentées en partie 3 et le choix des différentes hypothèses, pour les distances d'approvisionnement notamment, est discuté en partie 4.

\section{Résultats : analyse des performances environnementales d'une extension sur la mer et conséquences des choix logistiques et techniques}

Les résultats de l'analyse de contribution par étape des scénarios 1 et 2 sont fournies figure 3 (en couleurs unies pour le scénario 1 performant environnementalement et en couleurs pointillées pour le scénario 2 non optimisé). Pour chaque catégorie d'impact (en abscisse), les résultats sont fournis en pourcentage du scénario le plus impactant.

Pour des questions de lisibilité de la figure, certaines catégories d'impact de la méthode ILCD (toxicité humaine cancérigène, eutrophisation terrestre et radiations ionisantes E) ont été supprimées, car leurs résultats étaient redondants avec ceux d'autres catégories d'impact (respectivement toxicité humaine non cancérigène, eutrophisation d'eau douce et radiations ionisantes $\mathrm{HH}$ ). 


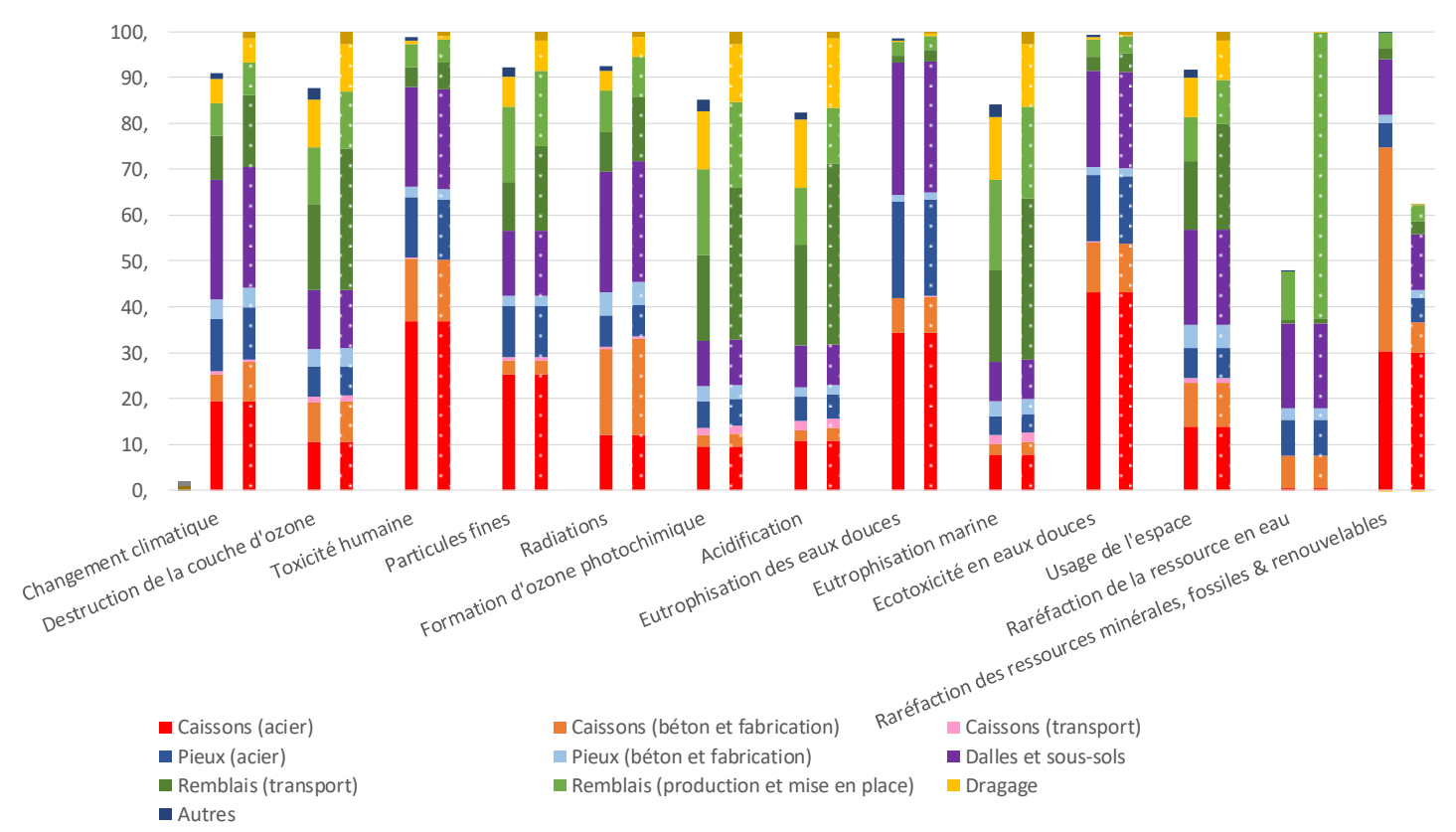

Figure 3. Impacts environnementaux des scénarios 1 (couleurs unies) et 2 (pointillées) par étapes et en pourcentage du scénario le plus impactant pour chaque catégorie.

Le scénario 1 montre un avantage environnemental pour l'ensemble des catégories d'impact quantifiées (-11,7\% de gain en moyenne), à l'exception de la raréfaction des ressources minérales et fossiles, liée à l'usage de ciment fortement substitué (CEM V) dans les caissons. Les bénéfices sont particulièrement importants sur la raréfaction de la ressource en eau, du fait qu'on utilise de l'eau de mer pour le lavage des matériaux de remblais dans le scénario 1 optimisé. L'optimisation des transports de matériaux de carrières permet également des gains importants pour la plupart des catégories d'impact, et notamment celles en lien avec la combustion de carburant (changement climatique, destruction de la couche d'ozone, émission de particules fines, formation d'ozone photochimique). Sur ces quatre catégories d'impact, l'optimisation des approvisionnements conduit à une diminution moyenne de $41 \%$ des impacts liés au transport des remblais.

Parmi les étapes de la fabrication de l'infrastructure maritime qui engendrent un fort impact sur l'environnement, y compris après optimisation de l'ouvrage, on retrouve notamment la production d'acier. Cela concerne les aciers du béton armé des caissons, des pieux, des dalles et des sous-sols de l'ouvrage. Pour ce qui concerne les autres hypothèses d'optimisation, l'impact sur les performances globales de l'ouvrage sont plus mitigées. L'usage de ciments partiellement substitués (CEM II pour le béton des pieux ou CEM V dans le béton des caissons) ne permet qu'un gain assez modeste, au vu de la relativement faible incidence des bétons en eux-mêmes sur les performances globales. 


\section{XVI'èmes Journées Nationales Génie Côtier - Génie Civil \\ Le Havre 2020}

\section{Limites de l'étude et d'applicabilité des solutions les plus performantes}

Dans notre cas d'étude, les zones portuaires industrielles se situent à plusieurs centaines de kilomètres du site de construction de l'extension (Toulon et Marseille). Cette problématique est certainement généralisable aux autres cas d'extensions en mer à usage d'habitation. Il s'agit d'infrastructures extrêmement coûteuses en investissement (plusieurs milliards d'euros), et de ce fait, leur localisation géographique est a priori limitée à des zones privilégiées, à l'écart des activités industrielles. L'usage de matériaux locaux est alors rendu difficile, car les infrastructures industrielles de stockage, de production, de chargement et déchargement s'avèrent limitées sur place.

La question de la temporalité joue également un rôle important dans la prise de décision concernant les approvisionnements en matériaux de remblais. En effet, sans contrainte sur la durée du chantier, on pourrait assez facilement imaginer un approvisionnement $100 \%$ local (sous réserve que la localité soit pourvue de carrières avoisinantes). Cependant, cela aurait pour conséquence un allongement significatif du temps de construction (d'au moins 19 mois dans notre cas d'étude). Au vu des investissements de tels projets, ces rallonges de temps ne sont pas envisageables par les investisseurs, même lorsqu'un approvisionnement local est techniquement possible. Par ailleurs, la dépose en mer de remblais arrivés par camion suppose la possibilité de chargements directement depuis le bord de mer, sur des navires de petite taille, ce qui complexifie la mise en œuvre et l'opérationnalité de cette solution.

Parmi les différentes hypothèses d'optimisation envisagées, certaines pourraient également être rendues difficiles par des contraintes de site. Par exemple, l'hypothèse d'un lavage à l'eau de mer après déchargement est soumise à la possibilité de réaliser cette opération sur le littoral. A défaut, ce lavage pourrait se faire sur des bateaux, ce qui est techniquement envisageable, mais impliquerait un changement de pratiques.

En dehors de ces verrous logistiques, d'autres pistes d'optimisation apparaissent au vu des résultats de cette analyse. La quantification de leurs avantages environnementaux fera l'objet d'un prochain article (travaux en cours par les auteurs). Il apparaît notamment important de tester les quantités d'acier (inox notamment) par rapport à la durabilité de l'ouvrage : jouer sur les épaisseurs d'enrobage semblerait plus pertinent au vu des impacts environnementaux des aciers et aciers inox. En effet, les ratios sont de $149 \mathrm{~kg}$ d'acier haute adhérence (acier d'armature carboné classique) et $47 \mathrm{~kg}$ d'acier inoxydable par $\mathrm{m}^{3}$ de béton armé dans les caissons. Pour les pieux, ce sont $136 \mathrm{~kg}$ d'acier d'armature qui sont utilisés par $\mathrm{m}^{3}$ de béton armé et $156 \mathrm{~kg}$ pour les tubages. Il apparaît également opportun de tester l'utilisation de matériaux de substitution plutôt que de matériaux de carrière pour les matériaux de remblais hydraulique et de remplissage des caissons. N'étant pas portants, ces remblais ont des spécifications moins contraignantes, bien que devant respecter des critères de compacité, de non liquéfaction, d'homogénéité, de granulométrie et être exempts de fines. 


\section{Thème 4 - Ouvrages portuaires, offshore et de plaisance}

\section{Conclusions}

Sur la base de notre cas d'étude d'extension sur la mer par réalisation d'un terre-plein et d'une digue d'enclôture en caissons en béton armé, il a été montré l'importance des choix logistiques sur les performances environnementales d'une telle infrastructure. Les contraintes liées au site influent très fortement sur l'applicabilité des optimisations : disponibilité de matériaux de carrière à proximité, autorisations d'exploitation par rapport aux besoins locaux, possibilité de stockage temporaire d'une partie des matériaux pour permettre un temps de travaux acceptable, choix politiques et infrastructures de circulation des camions sur la zone. Par ailleurs, l'écoconception en amont des projets et l'anticipation des besoins en matériaux sont des paramètres clés pour rendre possible l'optimisation des approvisionnements et la mobilisation des ressources locales, en accord avec les potentialités de la région. En l'absence d'anticipation et du fait de contraintes liées au site, la part des transports de matériaux s'avère générer un impact important sur l'environnement à l'échelle de tels projets.

Remerciements : Les auteurs remercient la Principauté de Monaco pour le financement de ces travaux de recherche. Ils remercient également la Mission Urbanisation en Mer de la Principauté de Monaco pour son support technique, ainsi que Bouygues Construction et la SAM de l'Anse du Portier pour la fourniture des données techniques ayant permis le dimensionnement de l'étude.

\section{Références bibliographiques}

ARISTAGHES P., BLANCHET V., COMPTE D., GREVOZ A., QUENOUILLE B., TELES M., WEULERSSE A. (2018). L'extension en mer de l'Anse du Portier à Monaco. Journées Nationales Génie Côtier - Génie Civil, La Rochelle, pp 409-420. https://doi.org/10.5150/jngcgc.2018.046

BRGM (2000). Schéma départemental des carrières des Alpes-Maritimes. 172 p. http://www.paca.developpement-durable.gouv.fr/IMG/pdf/SDC06_rapport-r_cle2b1476.pdf

ISO 14044 (2006). Environmental Management - Life Cycle Impact Assessment Requirements and guidelines. $46 \mathrm{p}$.

MEA -Millenium Ecosystem Assessment- (2005). Chapitre 19 Coastal Systems. In: Synthesis report. Island, Washington, DC., pp 513-549.

MEINESZ A., BLANFUNE A., CHANCOLLON O., JAVEL F., LONGEPIERRE S., MARKOVIC L., DE VAUGELAS J., GARCIA D. (2013). Côtes méditerranéennes françaises: inventaire et impacts des aménagements gagnés sur la mer. Ed. Lab. ECOMERS, Université Nice Sophia Antipolis. 153 p. www.medam.org

MINEO-KLEINER L., MEUR-FEREC C. (2016). Relocaliser les enjeux exposés aux risques côtiers en France: points de vue des acteurs institutionnels. Revue VertigO. Vol. 16, pp 1-32. https://doi.org/10.4000/vertigo.17656 